\title{
Clinical Audit Project Report
}

\section{-Audit of the Palliative Care Practitioners' Adherence to WHO Guidelines in Managing Patients' Pain at Cancer Diseases Hospital, Lusaka, Zambia}

\author{
Patience Mbozi $^{*}\left(\mathbb{D}\right.$, Cathy Ratcliff $^{2}$ \\ ${ }^{1}$ Ministry of Health, Cancer Diseases Hospital, Lusaka, Zambia \\ ${ }^{2}$ Independent Scholar and Director of International Programmes, EMMS International, Scotland, UK \\ Email: patiencembozi@gmail.com
}

How to cite this paper: Mbozi, P. and Ratcliff, C. (2019) Clinical Audit Project Report-Audit of the Palliative Care Practitioners' Adherence to WHO Guidelines in Managing Patients' Pain at Cancer Diseases Hospital, Lusaka, Zambia. Health, 11, 1631-1643. https://doi.org/10.4236/health.2019.1112124

Received: November 21, 2019

Accepted: December 27, 2019

Published: December 30, 2019

Copyright $\odot 2019$ by author(s) and Scientific Research Publishing Inc. This work is licensed under the Creative Commons Attribution International License (CC BY 4.0).

http://creativecommons.org/licenses/by/4.0/

\begin{abstract}
Appropriate adherence to World Health Organisation (WHO) pain management guidelines is vital in palliative care centres as it promotes the comfort of patients who are experiencing pain and it improves their quality of life. WHO (1996) highlighted the use of the WHO analgesic ladder guideline. This "analgesic ladder" proposes that after proper assessment with an appropriate pain assessment tool, patients in mild pain should be given non-opioids plus or minus adjuvants (including anticonvulsants and steroids); patients in moderate pain should be given weak opioids plus or minus non-opioids plus or minus adjuvants; and patients in severe pain should be given strong opioids plus or minus non-opioids plus or minus adjuvants as per the WHO pain ladder. The audit project was focused on assessing the palliative care practitioners' (PCPs) adherence to WHO guidelines in managing their patients' pain at Cancer Diseases Hospital (CDH). 15 participants were involved in the study and the data collection method used was a cross-sectional study in which the auditor observed PCPs, with an observation checklist comparing practice with WHO analgesic ladder guidelines. The result revealed that most of the PCPs were not meeting $80 \%$ of the standard set in the specific objectives for the audit. 7 (47\%) PCPs were scoring the pain level before analgesia administration, whereas 8 (53\%) PCPs were not scoring the pain level before analgesia administration. On the other hand, $47 \%$ (7 PCPs) were adhering to the WHO ladder of pain management, but $53 \%$ (8 PCPs) were not adhering to the WHO ladder of pain management. Therefore, effective adherence to the WHO analgesic ladder practice is still lacking in the PCPs at $\mathrm{CDH}$. The percentage of PCPs who were not scoring the pain and not adhering to the WHO pain ladder guidelines was high, which is 8 (53\%). Therefore, a re-audit is recommended to find out if levels of adherence have improved or not.
\end{abstract}




\section{Keywords}

Palliative Care Practitioners, Pain Assessment, Pain Management, Adherence, WHO Analgesic Ladder

\section{Introduction}

The International Association for the Study of Pain (IASP) defines pain as "an unpleasant sensory and emotional experience associated with actual or potential tissue damage, or described in terms of such damage" (IASP 1994) [1]. Adherence to the World Health Organisation (WHO) pain management guidelines is vital in palliative care, as it promotes the comfort of patients who are experiencing pain and improves their quality of life.

Awareness of palliative care practitioners (PCPs) of cancer pain and good working relationships among them is crucial for optimal pain management. Pain is a distressing symptom in palliative care and it should be effectively and efficiently managed. WHO (1996) indicates that being free of cancer pain is a basic human right, and further recommends that healthcare workers must use and adhere to the 3-step analgesic ladder guidelines in managing pain [2]. Moreover, one palliative care principle stipulates that healthcare workers should work towards improving the quality of life of patients and their families. Thus, the audit project was focused on how cancer pain is managed at Cancer Diseases Hospital $(\mathrm{CDH})$ using the WHO ladder guidelines. $\mathrm{CDH}$, in Lusaka, the capital of Zambia, is the only cancer hospital in Zambia. Therefore, it receives patients with all types of cancers from throughout Zambia, and at times even from neighbouring country Malawi.

Chow et al. (2010) articulated that pain remains an agonizing symptom in patients with advanced cancer and continues to be under-treated globally [3]. Granau et al. (2006), Hermann et al. (1986), and Schechter et al. (1986) also found out that, due to the obvious humanitarian reasons for treating pain, there is evidence that under-treatment of pain can lead to persistent pain, alterations in nociceptive processing and emotional and psychological complications [4] [5] [6]. Liu (1995) and Wasylak et al. (1990) reported that well-managed pain, on the other hand, is associated with faster recoveries, fewer complications and decreased use of healthcare resources [7] [8].

\subsection{Problem Statement}

According to WHO (2004), approximately $25 \%$ of HIV/AIDS patients and $80 \%$ of cancer patients suffer pain in the terminal phase of the illness [9]. WHO (1996) declared that opioid analgesic medication is the mainstay of pain treatment in cancer patients, according to WHO's guidelines for cancer pain relief [2].

Indeed, lack of proper adherence to the WHO analgesic ladder guidelines may impact negatively on patients' pain management, because a patient with severe 
pain who is not properly managed with opioids may not be comfortable and pain-free, which may in turn disturb their quality of life. Hence the audit set out to establish practice against standards set by World Health Organisation.

WHO (2004) adds that dissemination of proper pain control education has not prevented some cancer patients from suffering debilitating pain because of their own fears about narcotic addiction or their health workers' misunderstanding of appropriate pain medicine dosing [10]. Palliative care patients are many and inevitably suffer pain, but effective pain management may be quite limited. It has been observed at $\mathrm{CDH}$ that most cancer patients report for treatment very late, mostly due to financial constraints and long distances that they have to travel to Lusaka.

\subsection{Justification}

Cancer remains a major public health problem, and the control of end-of-life pain continues to be a challenge even in the face of major advancements in pain control methodology.

In a study by Bernardi et al. (2007) and McMillan et al. (2000), knowledge deficits on pain management in clinicians were found, and healthcare workers' (nurses') lack of knowledge may limit their advocacy for patients' proper pain management [11] [12].

Some PCPs possess some knowledge of pain management, but do not assess and manage the patients' pain at all; some prescriptions of pain medication may be done according to what the PCP presumes the patient is feeling.

In addition, most clinicians do not know how to manage pain effectively, despite having an idea of the WHO guidelines. Some may not believe patients' reports of pain. This was evidenced by Xue et al. (2007), who found that $59 \%$ of medical oncology nurses and $49 \%$ of gynaecological oncology nurses believed that patients under-reported the amount of pain experienced [12]. Despite this, most patients who come with advanced cancers suffer a great deal because of the advanced stage of their cancer.

Therefore the audit sought to identify existing practices which would inform $\mathrm{CDH}$ management and help strengthen or improve this healthcare practice so that patients' pain is properly managed to improve their and their families' quality of life.

\subsection{Literature Review}

Much is already known about pain management globally. This literature review highlights different clinical audit results on pain assessment and management practices. Existing information on management of pain is discussed under the following subthemes: use of analgesic ladder; health professionals' attitudes to prescription of opioids; and knowledge of types of pain.

The $\mathrm{CDH}$ has adopted the WHO guidelines for management of cancer pain because only cancer patients who suffer a great deal of pain are seen at the hospital. Portenoy (1989) deliberates that pain affects most patients with advanced cancer disease [13]. 


\section{Use of analgesic ladder}

For effective management of cancer pain, the WHO ladder approach was found effective and safe in $70 \%-90 \%$ of advanced cancer patients (Zech et al. 1995) [14]. However, Mayor (2000) and Pearl (1998) studies indicate that over 50\% of cancer patients worldwide do not receive adequate pain relief or are untreated and the unrelieved cancer pain persists as a significant public health concern [15] [16].

Ha konsen et al. (2008) also conducted an audit in pain management, and revealed that the highest adherence to the WHO analgesic ladder guidelines was for criteria relating to continuous analgesic drugs and to intermittent pain, and the measured adherence to WHO guideline was good (intermediate to high) [17].

They further stipulated that, although patients on by-the-clock analgesia received the recommended analgesic combination regimens without exceeding maximum dose or dose interval, some patients received analgesics corresponding to an inappropriate step of the WHO ladder. Ferrel et al. (1999) discuss that such inappropriate use of the analgesic ladder is a common problem in cancer pain management [18].

Rana et al. (2011) indicated that assessment and management of cancer pain is a crucial skill, which requires active listening and eye contact with the patient. Proper history-taking and physical examination are key to success [19]. WHO (1996) further indicated that patients need to receive appropriate analgesic treatment in a proactive manner based on the degree of pain they experience [2].

Zhu et al. (2012) also conducted an audit of pain assessment and pain management practices among hospital staff [20]. Interestingly this audit revealed that significantly higher rates of pain assessments, and more frequently administered pain management interventions, with the use of multimodal analgesia and continuous opioid infusions. Fewer patients experienced pain, and pain intensity was significantly lower.

\section{Health professionals' attitudes to prescription of opioids}

Thota et al. (2011) conducted an audit on opioid-prescribing practices in chronic cancer pain in a tertiary care pain clinic, and demonstrated under-treatment and under-dosing of opioid analgesics in a cancer pain clinic [21]. They found that the prescribed analgesic regimes were not adhering to the WHO guidelines, which recommend choice of oral analgesics according to the intensity of pain (mild, moderate and severe) (WHO 1996) [2]. Further their study revealed that strong opioids were not prescribed despite patients reporting severe pain. Overall, the study showed that only 93 of 184 patients with severe pain received morphine. However, 73 received an appropriate dose with an appropriate dosing interval.

Mercadante and Salvaggio (1996), Rajagopal et al. (2001) and Vanegas et al. (1998) revealed several factors contributing to the inappropriate use of opioid analgesics in cancer pain, such as insufficient education of doctors and nurses, exaggerated concerns about the risks of abuse and diversion of opioids, fear of 
side effects, and inappropriate attitudes among physicians, patients and family members of cancer patients [22] [23] [24]. Fujimoto (2001) added that existing national legislations and policies can be impediments to adequate opioid use [25].

\section{Knowledge of types of pain}

The WHO (2004) accentuates that the physician should know exactly the nature and type of pain and, accordingly, adjuvant agents should be incorporated in the treatment plan [9]. The degree of pain relief should be documented at regular time intervals. Similarly, Grond et al. (1996) emphasised that systematic and thorough initial assessment of pain is essential for treatment success [26]. Rana et al. (2011) further highlighted that pain management is considered adequate when there is congruence between patients' reported level of pain and the appropriateness of the prescribed analgesic drug [19].

\subsection{Standards}

\section{WHO standards of pain management}

$\mathrm{CDH}$ adopted the WHO standards of pain management; therefore the audit was based on WHO standards.

WHO (1996) highlighted the use of the "WHO analgesic ladder", simple but effective guidance on the management of pain [2]. This "analgesic ladder" proposes the following after proper assessment with an appropriate pain assessment tool: patients in mild pain should be given non-opioids plus or minus adjuvants (including anticonvulsants and steroids); patients in moderate pain should be given weak opioids plus or minus non-opioids plus or minus adjuvants; and patients in severe pain should be given strong opioids plus or minus non-opioids plus or minus adjuvants as per the WHO pain ladder (see Figure 1). Importantly, drugs should be given orally wherever possible and regularly, rather than waiting for pain to break through.

\subsection{Audit Question}

Do palliative care practitioners adhere to WHO guidelines in managing patients' pain?

\section{Objectives}

- To assess if $80 \%$ of palliative care practitioners at $\mathrm{CDH}$ are scoring patients' pain;

- To find out if $80 \%$ of PCPs at CDH are adhering to the WHO analgesic ladder in managing patients' pain.

\section{Methodology}

This chapter highlights various aspects of the approach used during the audit.

\subsection{Study Area}

Cancer Diseases Hospital in Lusaka District, the capital of Zambia, is the only cancer hospital and offers services to cancer patients only. 


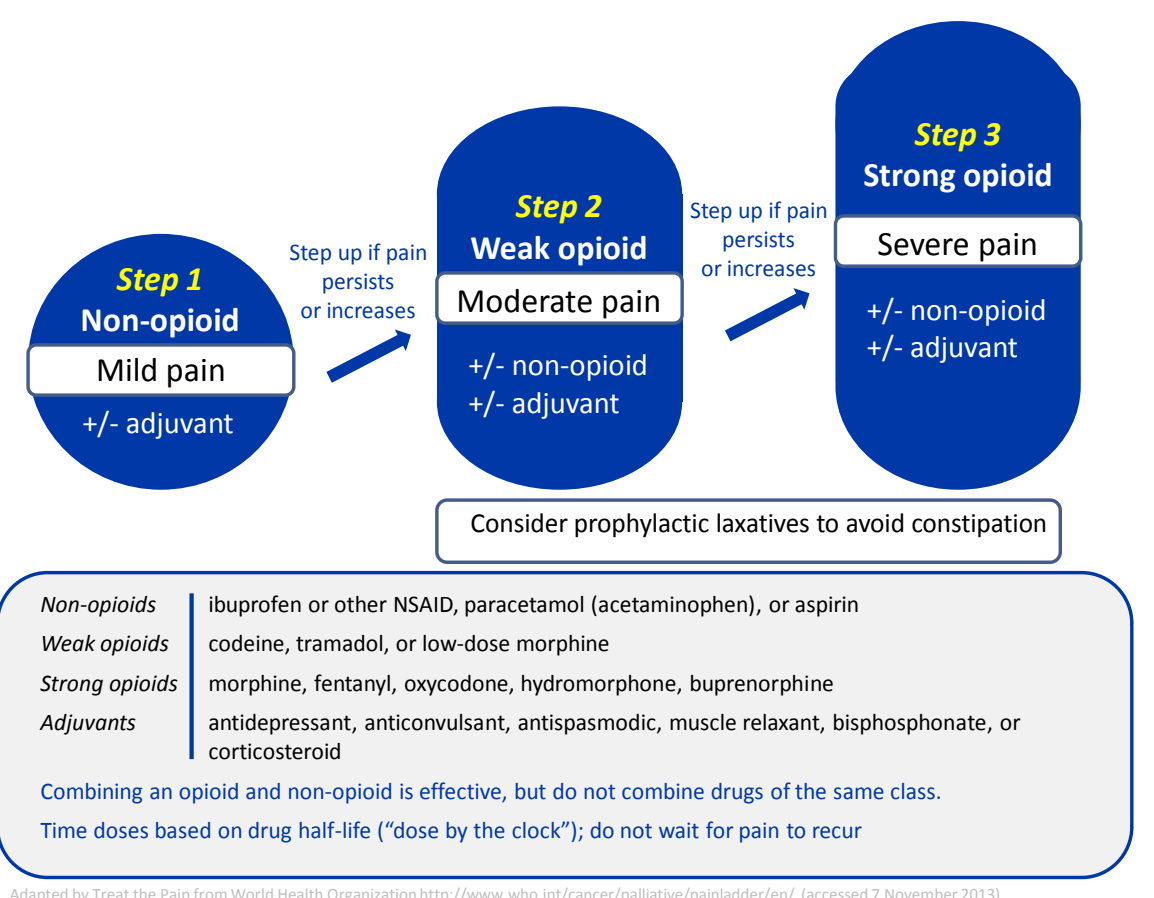

Figure 1. Adapted from World Health Organization (2004) three-step "ladder" for cancer pain relief in adults.

\subsection{Audit Design}

This was a cross-sectional study where the auditor observed PCPs, using an observation checklist to compare practice with WHO analgesic ladder guidelines.

\subsection{Audit Population}

The audit comprised of 15 PCPs who participate in pain assessment and management at Cancer Diseases Hospital.

\subsection{Inclusion Criteria}

Inclusion criteria in the audit comprised of PCPs who have participated in pain assessment and management at $\mathrm{CDH}$ for at least one year.

\subsection{Exclusion Criteria}

New staff and those unwilling to participate in the audit were not included in it.

\subsection{Audit Population-Sample Size}

15 PCPs (6 medical practitioners and 9 nurses) were observed.

\subsection{Sampling Procedure}

Purposive sampling method was used as it was easy to use and convenient.

\subsection{Data Collection}

Data was collected through an observation checklist. 


\subsection{Data Collection Procedures}

The purpose of the study was explained to all PCPs and audit participants at $\mathrm{CDH}$. Completed data collection tools were collected and kept in a safe place. The exercise was done until the desired sample size was obtained. Observations of PCPs as they tended to patients were noted each day.

\subsection{Data Processing Analysis and Interpretation}

Analysis of data collected was done manually, using tally sheets. The data was checked for accuracy, consistency and completeness.

\subsection{Ethical Considerations}

The auditor sought ethical approval from hospital administration to conduct the audit. On the observational checklist, anonymity for PCPs and patients was permitted. No PCP's name was indicated anywhere in the data collection sheet. Informed consent was taken from the PCPs before they fill in the questionnaire. Consent was obtained from all participants and the audit ensured that the rights of the participants were protected and confidentiality was maintained.

\subsection{Limitations of the Audit}

The number of PCPs reviewed may not be enough from which to generalise.

Incomplete patient records of interventions on pain assessment and management. Financial constraints delayed the collection of data and other information. Inadequate time allocated for the audit.

Unwillingness of some PCPs to participate, in case they were identified as lacking pain assessment and management skills.

\subsection{Utilisation and Dissemination of Results}

The audience of the Executive Director of CDH will be sought and the findings of the audit and the audit report will be presented. Recommendations to improve practice will be presented in a clinical presentation, to highlight the problem and possible solutions to clinical staff, so that practice can be improved. The audit report will be submitted to the Head of Clinical Care and all heads of departments.

\section{Data Presentation and Analysis}

\subsection{Introduction}

This chapter displays the presentation and analysis of the audit findings. 15 PCP participants were involved in the study; the sample included 09 females and 06 males, comprised of doctors and nurses.

\subsection{Data Presentation}

See Table 1 and Figures 2-3. 
Table 1. Performance of PCPs (n-15) in adherence to pain management guidelines using WHO ladder at $\mathrm{CDH}$.

\begin{tabular}{|c|c|c|}
\hline SKILL & Number of PCPs & Percentage of PCPs \\
\hline Scored pain before administration of pain medication & 7 & $47 \%$ \\
\hline $\begin{array}{l}\text { Give patients with mild pain step } 1 \text { analgesia such as } \\
\text { paracetamol and non-steroidal anti-inflammatory drugs, } \\
\text { with or without adjuvant drugs }\end{array}$ & 9 & $60 \%$ \\
\hline $\begin{array}{l}\text { Give patients with moderate pain step } 2 \text { analgesia such as } \\
\text { tramadol and codeine phosphate, with or without adjuvant } \\
\text { drugs }\end{array}$ & 10 & $67 \%$ \\
\hline $\begin{array}{l}\text { Give patients with severe pain analgesia such as morphine, } \\
\text { with or without adjuvant drugs }\end{array}$ & 13 & $87 \%$ \\
\hline $\begin{array}{l}\text { Are actually practicing adherence to WHO ladder } \\
\text { guidelines of managing pain }\end{array}$ & 8 & $55 \%$ \\
\hline Properly evaluate pain medication & 5 & $33 \%$ \\
\hline $\begin{array}{l}\text { Are knowledgeable of the drugs involved at each step of } \\
\text { the WHO ladder }\end{array}$ & 12 & $80 \%$ \\
\hline
\end{tabular}

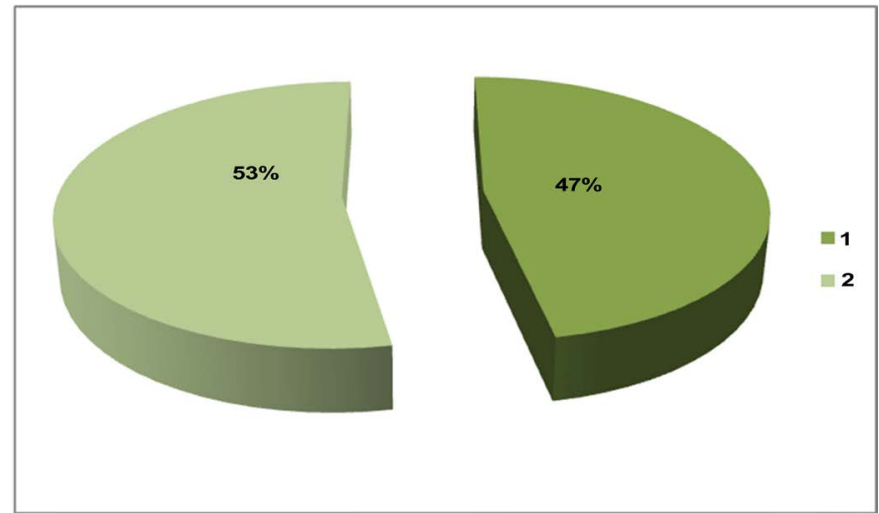

Figure 2. PCPs who scored pain before administration of pain medication. KEY: 1) PCPs 7 (47\%) scored the pain level before analgesia administration; 2) PCPs 8 (53\%) did not score the pain level before analgesia administration.

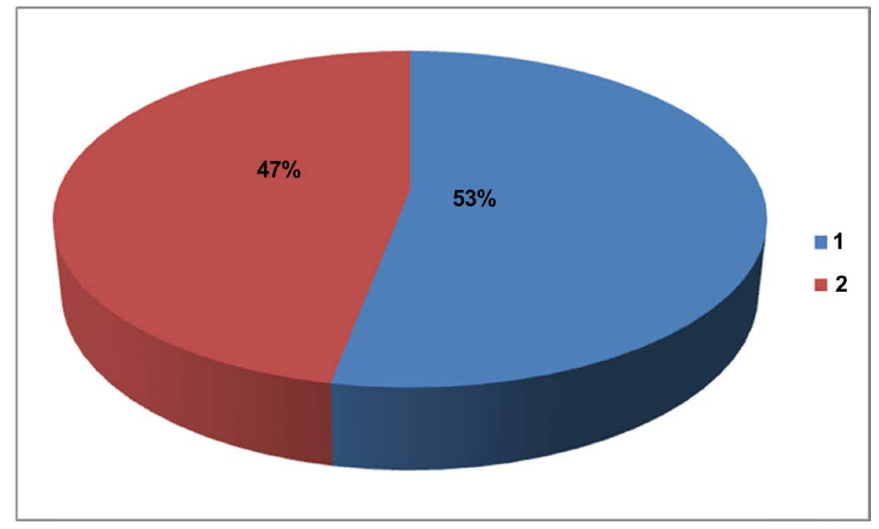

Figure 3. Adherence of PCPs (n-15) to WHO ladder guidelines in pain management. KEY: 1) Blue 53\% (8 PCPs) not adhering to the WHO ladder in pain management; 2 ) Red $47 \%$ (7 PCPs) adhering to the WHO ladder in pain management. 


\subsection{Data Analysis}

Data analysis was done manually by tallying using tally sheets.

Almost all PCPs know the drugs involved at each step of the WHO ladder.

$12(80 \%)$ and 7 (47\%) scored pain before administration of pain medication (see Figure 2, Figure 3 and Table 1).

PCPs who gave patients with mild pain step 1 analgesia such as paracetamol, non-steroidal anti-inflammatory drugs with or without adjuvant drugs $9(60 \%)$ (see Table 1).

PCPs who give patients with moderate pain step 2 analgesia such as tramadol, codeine phosphate, with or without adjuvant drugs 10 (67\%) (see Table 1).

PCPs who give patients with severe pain analgesia such as morphine, with or without adjuvant drugs 13 (87\%) (see Table 1).

PCPs who practised adherence to WHO ladder guidelines of managing pain 7 (47\%) (see Figure 2, Figure 3 and Table 1)

A good attitude towards patients and proper record-keeping is vital in pain management. PCPs who practised proper evaluation of pain medication interventions are $5(33 \%)$.

\section{Discussion, Conclusions and Recommendations}

\subsection{Discussion of Audit Findings}

The 15 PCPs were assessed using an observation checklist.

\section{Level of knowledge}

The majority $12(80 \%)$ of PCPs in this audit had knowledge of the WHO analgesic ladder, especially the group of drugs which should be used at each step of the ladder. This may be because most training institutions and hospitals emphasize the importance of being knowledgeable in pain management using the WHO analgesic ladder.

\section{Pain scoring findings}

Fewer than half of PCPs 7 (47\%) were observed to be scoring pain levels before analgesia administration, but 8 (53\%) PCPs were not scoring the pain level before analgesia administration (see Figure 2). This could have been because most health workers are over-worked with a high patient case load and hence do not have time to sit down and assess patients. This correlates with [27] Cohen et al. (2003) clinical audit on pain assessment and management which revealed that pain intensity was recorded for only $53 \%$ and $57 \%$ of patients, respectively, and that reassessments after treatment interventions were not routinely performed for these patients.

\section{Adherence findings}

Adherence of PCPs to the WHO ladder was 7 (47\%), and 8 (53\%) of PCPs were not adherent (see Figure 2 and Figure 3). PCPs who were adherent were giving analgesia according to the severity of pain in relation to the WHO analgesic ladder standards. The percentage of PCPs who were not adherent is alarming as most PCPs have knowledge of the WHO analgesic ladder but are not explicitly managing the patients' pain according to the guidelines of the WHO analgesic ladder. Most PCPs also have knowledge of WHO guidelines of pain management. 
Some clinicians just ask patients on follow-up visits what pain-killers they have been taking, in order to prescribe the same drug, instead of re-assessing the pain and whether it needs a stronger analgesia or not. This practice has greatly affected adherence to the WHO analgesic ladder, because proper reassessments on subsequent visits are vital to enable the PCP to decide whether patients' pain is properly managed or increasing and whether they need a stronger pain-killer.

\section{Opioids prescription practices of PCPs}

A good number of PCPs 13 (87\%) gave morphine to patients with severe pain with or without adjuvant drugs. This contradicts McCaffery (1992) and Fujimoto (2001) studies which revealed that appropriate use of opioid analgesics in cancer pain management by physicians is inadequate for a variety of reasons, including insufficient knowledge of analgesic pharmacology, myths and misconceptions, beliefs, and attitudes (addiction and fear of adverse effects) [28] [25].

Fujimoto (2001) emphasised that medical practitioners are reluctant to use morphine for many reasons, such as exaggerated ideas about its poor safety profile and some thinking that prescribing morphine means admitting therapeutic defeat [25]. He reported that many healthcare workers find it hard to come to terms with the fact that treatment has failed and the disease is progressing, and the patient will die. Moreover, as physicians do not accept this, it is the patient who faces the consequences of unrelieved physical pain due to inadequate analgesia, coupled perhaps with a false hope of an eventual treatment response. Similarly, Forbes (2011) added that patients' attitudes also contribute to avoiding strong opioids, as many patients are concerned that taking strong opioids means their cancer is progressing coupled with fear of intolerable opioid side-effects [29].

\subsection{Conclusions}

The following conclusions were drawn from the study findings.

Most health workers 53\% (8) are not adhering to WHO standards of pain management, which is alarming for medical practice because it is not meeting the $80 \%$ of PCPs set in the audit objectives. On the other hand, 7 (47\%) were adhering to WHO guidelines. Moreover, 7 (47\%) PCPs were observed to have been scoring pain levels before analgesia administration, whereas 8 (53\%) PCPs were not scoring pain levels before analgesia administration (see Figure 2). This still does not meet the $80 \%$ standards set for the audit. Therefore, adherence to the WHO analgesic ladder practice is still lacking in PCPs at CDH.

\subsection{Recommendations}

The percentage of PCPs not adhering to the WHO pain ladder is high at 53\% (8) (see Figure 2 and Figure 3). Therefore a re-audit is recommended to complete the audit cycle, influence practice and find out if levels of adherence have improved.

$\mathrm{CDH}$ should continuously sensitise staff on pain assessment and management through continuous medical education and refresher courses. 
PCPs should re-assess pain every time patients come for refills of pain medication and on follow-up visits.

Pain assessment must be the fifth vital sign together with temperature, pulse, blood pressure and respiration, to ensure that patients in pain are identified early.

\subsection{Implications for Palliative Care Practice}

The audit study and result have huge implications for palliative care service delivery.

A few health workers who are not assessing and managing pain according to the WHO ladder accelerate patients' illnesses. Therefore these gaps must be dealt with by ensuring patients in pain are identified early, assessed appropriately and managed according to the guidelines set to improve patients' quality of life.

\section{Acknowledgements}

My God the Almighty for the gift of life and my medical career. My dear husband, friend Festus Simuunza and my sons Pascal, Nathanael, Jayson and Miguel Simuunza. Ministry of Health—Cancer Diseases Hospital.

\section{Conflicts of Interest}

The authors declare no conflicts of interest regarding the publication of this paper.

\section{References}

[1] International Association for Study of Pain (1994) ISAP Pain Terminology. http://www.iasp-pain.org/defsopen.html

[2] World Health Organization (1996) Cancer Pain Relief: With a Guide to Opioid Availability. 2nd Edition, World Health Organization, Geneva.

[3] Chow, E., Rosenbluth, R., Schofield, P. and Merrick, J. (2010) Advanced Cancer and Pain. Journal of Pain Management, 3, 3-4.

[4] Grunau, R.E., Holsti, L. and Peters, J.W.B. (2006) Long-Term Consequences of Pain in Human Neonates. Seminars in Fetal \& Neonatal Medicine, 1, 268-275. https://doi.org/10.1016/j.siny.2006.02.007

[5] Hermann, C., Hohmeister, J., Demirakca, S., Zohsel, K. and Flor, H. (2006) Long-Term Alteration of Pain Severity in School-Aged Children with Early Pain Experiences. Pain, 125, 278-285. https://doi.org/10.1016/j.pain.2006.08.026

[6] Schechter, N.L., Alen, D.A. and Hanson, K. (1986) Status of Pediatric Pain Control: A Comparison of Hospital Analgesic Usage in Children and Adults. Pediatrics, 77, 11-15.

[7] Liu, S., Carpenter, R.L. and Neal, J.M. (1995) Epidural Anaesthesia and Analgesia. Their Role in Postoperative Outcome. Anaesthesiology, 82, 1474-1506.

https://doi.org/10.1097/00000542-199506000-00019

[8] Wasylak, T.J., English, M.J.M. and Jeans, M.-E. (1990) Reduction of Postoperative Morbidity Following Patient Controlled Morphine. Canadian Journal of Anesthesia, 37, 726-731. https://doi.org/10.1007/BF03006529

[9] World Health Organization (2004) A Community Health Approach to Palliative 
Care for HIV/AIDS and Cancer Patients in Sub-Saharan Africa. World Health Organization, Geneva.

[10] Bernardi, M., Catania, G. and Tridello, G. (2007) Knowledge and Attitudes about Cancer Pain Management: A National Survey of Italian Hospice Nurses. Cancer Nursing, 30, E20-E26. https://doi.org/10.1097/01.NCC.0000265299.25017.24

[11] McMillan, S.T., Hagan, S., Laughlin, J. and Tabler, R. (2000) Knowledge and Attitudes of Nurses in Veterans' Hospitals about Pain Management in Patients with Cancer. Oncology Nursing Forum, 27, 1415-1423.

[12] Xue, Y., Schulman-Green, D., Czaplinski, C., Harris, D. and McCorkle, R. (2007) Pain Attitudes and Knowledge among RNs, Pharmacists, and Physicians on an Inpatient Oncology Service. Clinical Journal of Oncology Nursing, 11, 687-695. https://doi.org/10.1188/07.CJON.687-695

[13] Portenoy, R.K. (1989) Cancer Pain. Epidemiology and Syndromes. Cancer, 63, 22982307.

https://doi.org/10.1002/1097-0142(19890601)63:11<2298::AID-CNCR2820631140> 3.0.CO;2-A

[14] Zech, D.F., Grond, S., Lynch, J., Hertel, D. and Lehmann, K.A. (1995) Validation of World Health Organization Guidelines for Cancer Pain Relief: A 10-Year Prospective Study. Pain, 63, 65-76. https://doi.org/10.1016/0304-3959(95)00017-M

[15] Mayor, S. (2000) Survey of Patients Shows That Cancer Pain Still Undertreated. British Medical Journal, 321, 1309. https://doi.org/10.1136/bmj.321.7272.1309/b

[16] Pearl, J.D. (1998) Cancer Pain Management: Still a Public Health Issue. American Society of Anesthesiologists Newsletter, 62, 18-20.

[17] Ha Konsen, G.D., Strelec, P., Campbell, D., Hudson, S. and Loennechen, T. (2008) Adherence to Medication Guideline Criteria in Cancer Pain Management. Journal of Pain and Symptom Management, 37, 1006-1018. https://doi.org/10.1016/j.jpainsymman.2008.06.006

[18] Ferrel, B.R., Juarez, G. and Borneman, T. (1999) Use of Routine and Breakthrough Analgesia in Home Care. Oncology Nursing Forum, 26, 1655-1661.

[19] Rana, S.P.S., Gupta, R., Chaudhary, P., Khurana, D., Mishra, S. and Bhatnagar, S. (2011) Cancer Pain Management: Basic Information for the Young Pain Physicians. Indian Journal of Palliative Care, 17, 127-129. https://doi.org/10.4103/0973-1075.84533

[20] Zhu, L.M., Stinson, J., Palozzi, L., Weingarten, K., Hogan, M.E., Duong, S., Carbajal, R. and Campbell, F.A. (2012) Improvements in Pain Outcomes in a Canadian Paediatric Teaching Hospital Following Implementation of a Multifaceted, Knowledge Translation Initiative. Pain Research and Management, 17, 173-179. https://doi.org/10.1155/2012/586589

[21] Thota, R.S., Jain, P.N., Bakshi, S.G. and Dhanve, C.N. (2011) Opioid-Prescribing Practices in Chronic Cancer Pain in a Tertiary Care Pain Clinic. Indian Journal of Palliative Care, 17, 222-226. https://doi.org/10.4103/0973-1075.92340

[22] Mercadante, S. and Salvaggio, L. (1996) Cancer Pain Knowledge in Southern Italy: Data from a Postgraduate Refresher Course. Journal of Pain and Symptom Management, 11, 108-115. https://doi.org/10.1016/0885-3924(95)00147-6

[23] Rajagopal, M.R., Joranson, D.E. and Gilson, A.M. (2001) Medical Use, Misuse, and Diversion of Opioids in India. The Lancet, 358, 139-143. https://doi.org/10.1016/S0140-6736(01)05322-3

[24] Vanegas, G., Ripamonti, C., Sbanotto, A. and De Conno, F. (1998) Side Effects of Morphine Administration in Cancer Patients. Cancer Nursing, 21, 289-297. 
https://doi.org/10.1097/00002820-199808000-00011

[25] Fujimoto, D. (2001) Regulatory Issues in Pain Management. Clinics in Geriatric Medicine, 17, 537-551. https://doi.org/10.1016/S0749-0690(05)70085-9

[26] Grond, S., Zech, D., Diefenbach, C., Radbruch, L. and Lehmann, K. (1996) Assessment of Cancer Pain: A Prospective Evaluation in 2266 Cancer Patients Referred to a Pain Service. Pain, 64, 107-114. https://doi.org/10.1016/0304-3959(95)00076-3

[27] Cohen, M.Z., Easley, M.K., Ellis, C., et al. (2003) Cancer Pain Management and the JCAHO's Pain Standards: An Institutional Challenge. Journal of Pain and Symptom Management, 25, 519-527. https://doi.org/10.1016/S0885-3924(03)00068-X

[28] McCaffery, M. (1992) Pain Control. Barriers to the Use of Available Information. World Health Organization Expert Committee on Cancer Pain Relief and Active Supportive Care. Cancer, 70, 1438-1439.

https://doi.org/10.1002/1097-0142(19920901)70:3+<1438::AID-CNCR2820701536> 3.0.CO;2-W

[29] Forbes, K. (2011) Pain in Patients with Cancer: The World Health Organization Analgesic Ladder and Beyond. Journal of Clinical Oncology, 23, 379-380.

https://doi.org/10.1016/j.clon.2011.04.016

\section{List of Acronyms/Abbreviations}

AIDS-Acquired Immuno-Deficiency Syndrome

$\mathrm{CDH}-$ Cancer Diseases Hospital

HIV-Human Immunodeficiency Virus

IASP - International Association for the Study of Pain

PCPs-Palliative Care practitioners

WHO-World Health Organisation 